\title{
Localisation of a candidate anion transporter to the surface of the malaria parasite
}

\author{
Roselani I. Henry, Rowena E. Martin, Susan M. Howitt, Kiaran Kirk * \\ School of Biochemistry and Molecular Biology, The Australian National University, Canberra ACT 0200, Australia
}

Received 16 August 2007

Available online 29 August 2007

\begin{abstract}
PfSulP, encoded by the human malaria parasite Plasmodium falciparum, is a member of the sulphate permease family of anion transporter proteins. By transfecting the parasite with an epitope-tagged version of PfSulP, and detecting via western blot and indirect immunofluorescent assay microscopy, we show that PfSulP is localised to the surface of the intraerythrocytic parasite, where it is postulated to play a role in the flux of anions across the parasite plasma membrane.
\end{abstract}

(c) 2007 Elsevier Inc. All rights reserved.

Keywords: Plasmodium falciparum; Membrane; Anion; Transporter; Permease; SulP; Sulphate; Chloride

The malaria parasite, a unicellular eukaryote which, in the course of its complex lifecycle, invades the erythrocytes of its vertebrate host, is responsible for up to three million deaths and close to five billion episodes of clinical illness annually [1]. As it develops within the infected blood cell the parasite is reliant on membrane transport proteins to mediate the uptake of nutrients, the efflux of metabolic wastes, and the maintenance of ion homeostasis. Analysis of the genome of the human malaria parasite Plasmodium falciparum has led to the annotation of over a hundred different membrane transport proteins [2]. These proteins are of interest as potential drug targets in their own right, as well as for their possible role(s) in antimalarial drug resistance [3]. However, in all but a few cases, their subcellular localisation, substrate specificity, or physiological role(s) remain to be determined.

The transport of inorganic anions across cellular membranes can affect factors such as the $\mathrm{pH}$, volume and membrane potential of a cell, and in doing so can affect a vast array of cellular processes. The $P$. falciparum protein PF14_0679 (gi:23497754), designated here as PfSulP, is a member of the SulP (sulphate permease) family of second-

\footnotetext{
* Corresponding author. Fax: +61 261250313.

E-mail address: kiaran.kirk@anu.edu.au (K. Kirk).
}

ary active anion transporters and is a likely candidate for the transport of inorganic anions in the malaria parasite. The corresponding mRNA is expressed throughout the intraerythrocytic lifestages of the parasite [2]. Here we present a brief bioinformatic analysis of the PfSulP protein and report its cloning and subcellular localisation.

\section{Materials and methods}

Bioinformatics. A protein alignment of SulP family members, in which two proteins of the distantly related 'anion exchanger' (AE) family (NCBI database Accession Codes 114787 and 108883391) were included as an outgroup, was generated and edited in MacVector ${ }^{\mathrm{TM}}$ 7.1.1. Regions that could not be aligned unambiguously were excluded prior to analysis. A phylogenetic tree was estimated using the Neighbour-Joining method [4] and uncorrected ('p') amino acid distances. Ties in the tree were resolved randomly and a bootstrap analysis [5] was performed with 1000 pseudoreplicates.

The putative transmembrane topology of PfSulP was established from hydropathy plot analyses using the web-based programs TMHMM v 2.0 (CBS; Denmark), TMpred (EMBnet-CH), and PredictProtein [6], and from the analysis of a protein sequence alignment of the PfSulP family. The resulting figure was generated using the TOPO2 transmembrane protein display tool (SACS, UCSF; http://www.sacs.ucsf.edu/TOPO-run/ wtopo.pl).

Subcellular localisation. The subcellular localisation of PfSulP in human erythrocytes infected with mature trophozoite-stage $P$. falciparum (3D7) infected erythrocytes was investigated by transfecting parasites with 
a vector encoding a haemagglutinin (HA)-tagged form of the PfSulP protein (PfSulP-HA), with three sequential copies of the HA tag fused to the N-terminus. The full sequence of PfSulP was amplified from $P$. falciparum 3D7 cDNA using appropriate primer sets, and inserted into the vector $\mathrm{pDONR}{ }^{\mathrm{TM}} \mathrm{P} 2 \mathrm{R}-\mathrm{P} 3$ (Invitrogen) via a $\mathrm{BP}$ clonase recombination reaction. A transfection vector, in which expression of the HA-tagged protein is under the control of the 'chloroquine resistance transporter' promoter was generated using a vector set developed by van Dooren et al. [7] as described previously [8,9]. The construct was electroporated into 3D7 parasites as described by Wu et al. [10] and transfectants were selected with WR99210 (5 mM).

Expression of the HA-tagged protein was confirmed by Western blot analysis of protein samples prepared as described previously [11]. The proteins were separated on a denaturing gel, labelled with a mouse monoclonal anti-HA antibody (1/5000) and a goat anti-mouse IgG peroxidase conjugate secondary antibody (1/10,000; MP Biomedicals), and detected using a chemiluminescent peroxidase substrate.

For immunofluorescence assays parasitised erythrocytes were fixed and labelled using a protocol described elsewhere [11]. Primary antibodies [a mouse anti-HA antibody, and rabbit PfENT1 antisera (a kind gift from B. Ullman, Oregon Health and Science University)] were used at a 1 in 200 dilution. Secondary antibodies (AlexaFluor 488 goat anti-mouse and AlexaFluor 594 goat anti-rabbit; Molecular Probes) were used at a 1 in 1000 dilution. Nuclei were stained with DAPI. Fluorescence and DIC (differential interference contrast) images were collected using a Leica laser scanning confocal microscope with a $63 \times$ water lens.

\section{Results and discussion}

\section{Bioinformatics}

PlasmoDB predicts a six-exon structure for the PfSulP gene. However, examination of the genomic sequence surrounding the gene revealed an alternative model with a five-exon configuration, in which the fifth exon is longer (by 42 bases) than predicted. In PCR analyses using primer pairs specific for each gene model, and genomic DNA-free $P$. falciparum cDNA as template, only the coding sequence of the five-exon gene model was able to be amplified, consistent with this (five-exon) model representing the true coding sequence.

PfSulP bears good sequence similarity (BLASTP ' $E$ ' values $\geqslant \mathrm{e}^{-62}$ ) to bacterial members of the SulP family and a phylogenetic analysis (Fig. 1A) places PfSulP, together with SulP homologues from other apicomplexan species, within a large group containing bacterial homologues.

The SulP family is so-called because the first members to be characterised, from yeast and plants, were identified as $\mathrm{SO}_{4}{ }^{2-} / \mathrm{H}^{+}$symporters. However, it has since been demonstrated that SulP proteins can facilitate a variety of anion transport processes. Mammalian SulPs have been shown to catalyse anion exchange processes including, but not limited to, $\mathrm{SO}_{4}{ }^{2-} / \mathrm{HCO}_{3}{ }^{-}$exchange, $\mathrm{Cl}^{-} / \mathrm{HCO}_{3}{ }^{-}$exchange, $\mathrm{SO}_{4}{ }^{2-} / \mathrm{Cl}^{-}$exchange and $\mathrm{Cl}^{-} /$formate exchange. One cyanobacterial homologue ( $\operatorname{LtnT})$ has been implicated in nitrate transport [12] while another (BicA) has been shown to catalyse $\mathrm{HCO}_{3}^{-} / \mathrm{Na}^{+}$symport [13]. A bioinformatic analysis revealed that many bacterial SulP homologues are fused to homologues of the enzyme carbonic anhydrase [14], prompting the suggestion that they might transport $\mathrm{HCO}_{3}{ }^{-}$as their primary substrate. By contrast, a SulP
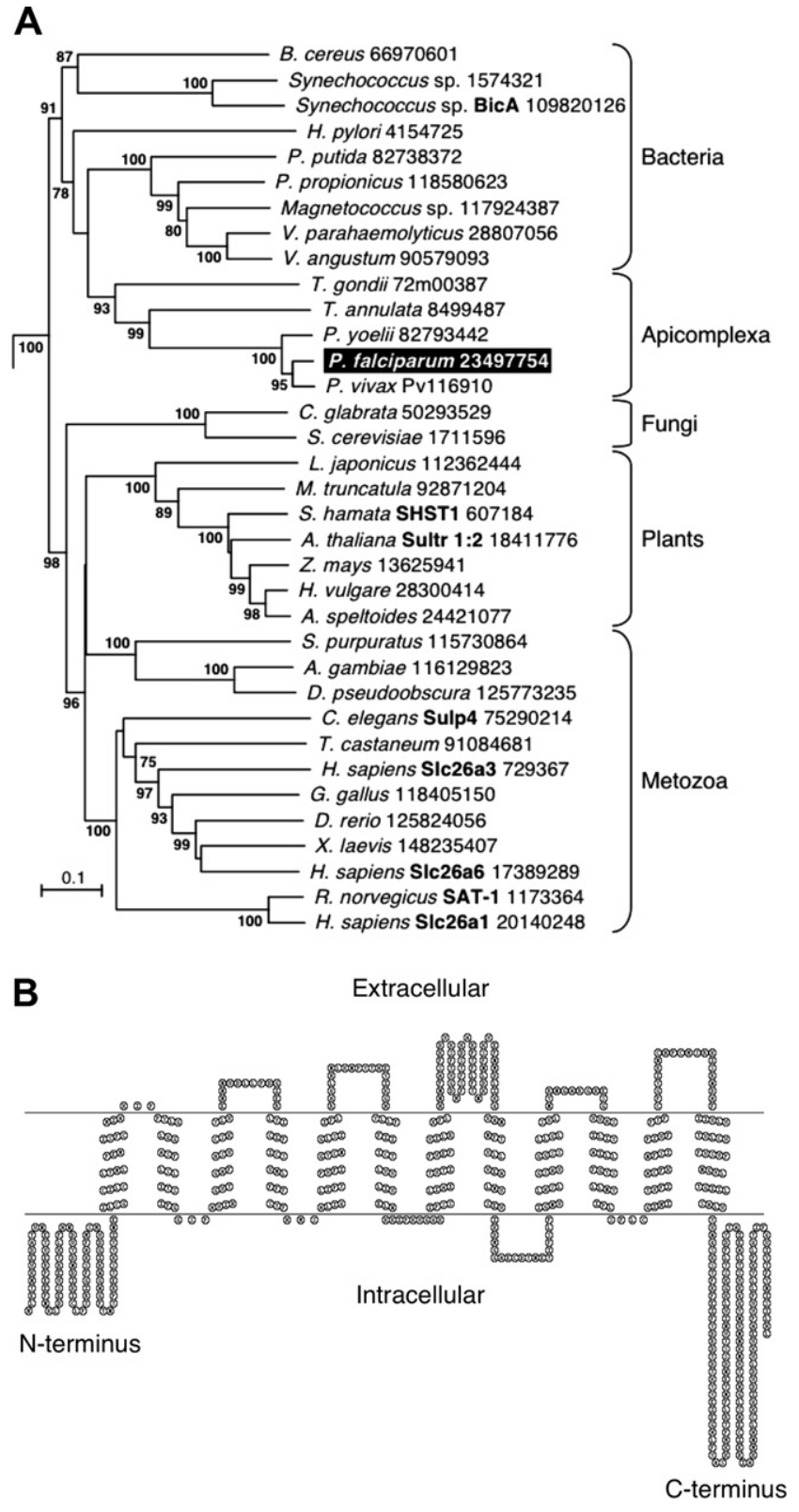

Fig. 1. The P. falciparum PfSulP protein. (A) A phylogenetic tree showing relationships between PfSulP (highlighted in black) and homologues in other organisms. Bootstrap scores of more than $70 \%$ are shown on the branches. The scale bar represents the number of substitutions per site for a unit branch length. (B) Proposed transmembrane topology of PfSulP, established from hydropathy plot analyses and from the analysis of a protein sequence alignment of the PfSulP family.

homologue from the bacterium Chloroflexus aurantiacus is fused to a domain of rhodanese, a thiosulphate:cyanide sulfotransferase [15], consistent with a role as a sulphate permease.

PfSulP is predicted, on the basis of hydrophobicity analysis, to have 12 transmembrane helices (Fig. 1B). The termini are predicted (on the basis of the 'positive inside' rule $[16,17])$ to reside on the cytosolic side of the membrane. The cytosolic C-terminus of PfSulP, like that in other SulP homologues, is large (approximately 
140 amino acids long). In many SulP proteins the C-terminus houses a STAS domain (Pfam 01740.12); i.e. a domain with similarity to a class of soluble bacterial proteins called antisigma-factor antagonists [18]. Although PfSulP has not been assigned a Pfam designation of this domain, a BLAST search conducted using only the Cterminus of PfSulP brought up numerous STAS domain proteins including STAS-containing SulPs and antisigmafactor antagonist proteins. The functional role of the STAS domain in SulP proteins has not yet been resolved; however, random mutagenesis of an Arabidopsis homologue has shown that the domain is important for both trafficking and function of the transporter [19].

\section{Subcellular localisation}

To investigate the localisation of PfSulP in the P. falciparum-infected human erythrocyte parasites were transfected with a vector encoding an HA-tagged form of the PfSulP protein, with three sequential copies of the HA tag fused to the $\mathrm{N}$-terminus. This approach has been employed previously to localise several other parasiteencoded transporters in the infected cell $[8,9]$.

Fig. 2A shows a Western blot of protein prepared from the transfected parasites and from a non-transfected control, probed with an anti-HA antibody. The protein preparation from the transfectants exhibited a single band that corresponded approximately to the predicted size of PfSulP-HA $(\sim 76 \mathrm{kDa})$. There was no band in the nontransfected control.

In order to determine the subcellular localisation of PfSulP, transfectant cells were fixed, immunostained with fluorophore-conjugated antibodies and observed by fluorescent microscopy. The upper panel of Fig. 2B shows the pattern of HA-associated fluorescence in the transfectants. Fluorescence was localised to the vicinity of the parasite surface. Some fluorescence was also observed around the parasite digestive vacuole; however this was probably due to the reflective properties of this compartment rather than HA-specific staining, as similar vacuole fluorescence was observed in non-transfected control cells (data not shown). No fluorescence was observed at the erythrocyte plasma membrane.

The lower panel of Fig. 2B shows the PfSulP-HAassociated fluorescence co-localised with fluorescently labelled PfENT1, a transport protein shown previously to reside on the plasma membrane of the parasite [20]. HA-associated fluorescence and PfENT1-associated fluorescence localised together at the parasite surface.

Attempts to gain insight into the transport function of PfSulP using the Xenopus laevis oocyte expression system proved unsuccessful. Oocytes injected with cRNA encoding PfSulP showed no significant change in the rate of uptake of ${ }^{36} \mathrm{Cl}^{-},{ }^{35} \mathrm{SO}_{4}{ }^{2-}$, or ${ }^{33} \mathrm{P}_{\mathrm{i}}$. Whether this was due to a lack of functional expression of the protein in the heterologous system, or due to an inappropriate choice of substrate was not investigated.

\section{Conclusion}

In summary, the PfSulP protein belongs to a family of anion transporters. An HA-tagged form of the protein localised to the surface of the parasite within trophozoite-infected erythrocytes, consistent with it being present on the parasite's plasma membrane. It is therefore a good candidate for playing a role in the movement of anions between the intraerythrocytic parasite and its host cell.
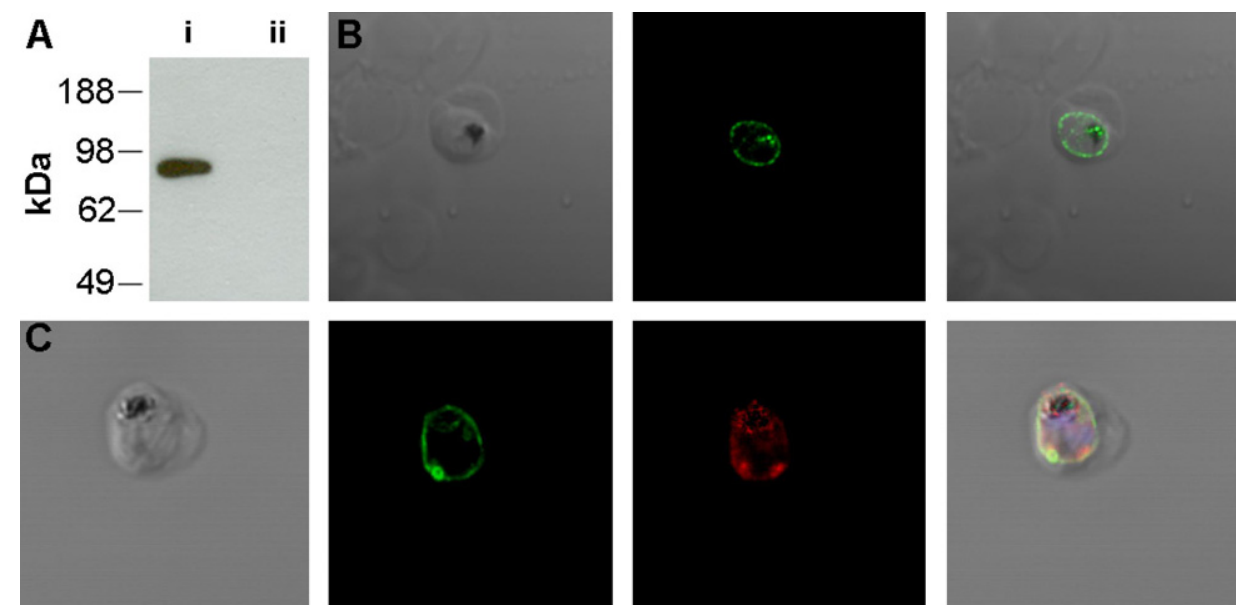

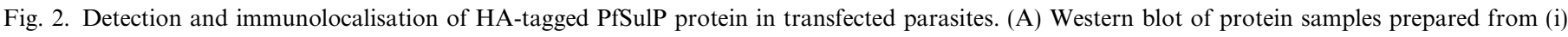

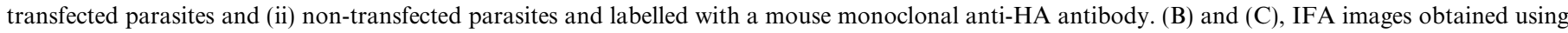

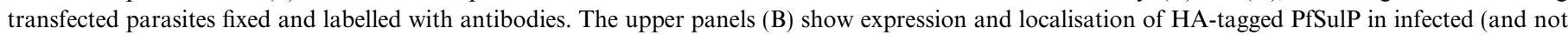

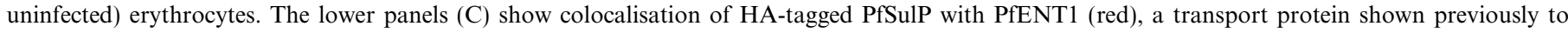

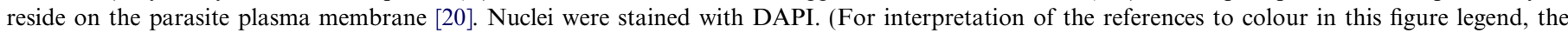
reader is referred to the web version of this article.) 


\section{Acknowledgments}

We are grateful to Kylie Mullin and Geoff McFadden (University of Melbourne) for helpful advice, to the Canberra Branch of the Australian Red Cross Blood Service for the provision of blood, to the ARC/NHMRC Research Network for Parasitology for a Researcher Exchange Award, and to the ARC and NHMRC for grant support.

\section{References}

[1] J.G. Breman, M.S. Alilio, A. Mills, Conquering the intolerable burden of malaria: what's new, what's needed: a summary, Am. J. Trop. Med. Hyg. 71 (2004) 1-15.

[2] R.E. Martin, R.I. Henry, J.L. Abbey, J.D. Clements, K. Kirk, The 'permeome' of the malaria parasite: an overview of the membrane transport proteins of Plasmodium falciparum, Genome. Biol. 6 (2005) R26.

[3] K. Kirk, Channels and transporters as drug targets in the Plasmodium-infected erythrocyte, Acta Trop. 89 (2004) 285-298.

[4] N. Saitou, M. Nei, The neighbor-joining method: a new method for reconstructing phylogenetic trees, Mol. Biol. Evol. 4 (1987) 406-425.

[5] J. Felsenstein, Confidence-limits on phylogenies - an approach using the bootstrap, Evolution 39 (1985) 783-791.

[6] B. Rost, G. Yachdav, J. Liu, The PredictProtein server, Nucleic. Acids Res. 32 (2004) W321-W326.

[7] G.G. van Dooren, M. Marti, C.J. Tonkin, L.M. Stimmler, A.F. Cowman, G.I. McFadden, Development of the endoplasmic reticulum, mitochondrion and apicoplast during the asexual life cycle of Plasmodium falciparum, Mol. Microbiol. 57 (2005) 405419.

[8] K.A. Mullin, L. Lim, S.A. Ralph, T.P. Spurck, E. Handman, G.I. McFadden, Membrane transporters in the relict plastid of malaria parasites, Proc. Natl. Acad. Sci. USA 103 (2006) 9572-9577.

[9] K.J. Saliba, R.E. Martin, A. Broer, R.I. Henry, C.S. McCarthy, M.J. Downie, R.J. Allen, K.A. Mullin, G.I. McFadden, S. Broer, K. Kirk,
Sodium-dependent uptake of inorganic phosphate by the intracellular malaria parasite, Nature 443 (2006) 582-585.

[10] Y. Wu, C.D. Sifri, H.H. Lei, X.Z. Su, T.E. Wellems, Transfection of Plasmodium falciparum within human red blood cells, Proc. Natl. Acad. Sci. USA 92 (1995) 973-977.

[11] C.J. Tonkin, G.G. van Dooren, T.P. Spurck, N.S. Struck, R.T. Good, E. Handman, A.F. Cowman, G.I. McFadden, Localization of organellar proteins in Plasmodium falciparum using a novel set of transfection vectors and a new immunofluorescence fixation method, Mol. Biochem. Parasitol. 137 (2004) 13-21.

[12] S. Maeda, C. Sugita, M. Sugita, T. Omata, Latent nitrate transport activity of a novel sulfate permease-like protein of the cyanobacterium Synechococcus elongatus, J. Biol. Chem. 281 (2006) 5869-5876.

[13] G.D. Price, F.J. Woodger, M.R. Badger, S.M. Howitt, L. Tucker, Identification of a SulP-type bicarbonate transporter in marine cyanobacteria, Proc. Natl. Acad. Sci. USA 101 (2004) 18228-18233.

[14] J. Felce, M.H. Saier Jr., Carbonic anhydrases fused to anion transporters of the SulP family: evidence for a novel type of bicarbonate transporter, J. Mol. Microbiol. Biotechnol. 8 (2004) 169-176.

[15] D. Bordo, P. Bork, The rhodanese/Cdc25 phosphatase superfamily. Sequence-structure-function relations, EMBO Rep. 3 (2002) 741-746.

[16] G.V. Heijne, The distribution of positively charged residues in bacterial inner membrane proteins correlates with the trans-membrane topology, EMBO J. 5 (1986) 3021-3027.

[17] J. Nilsson, B. Persson, G. von Heijne, Comparative analysis of amino acid distributions in integral membrane proteins from 107 genomes, Proteins 60 (2005) 606-616.

[18] L. Aravind, E.V. Koonin, The STAS domain-a link between anion transporters and antisigma-factor antagonists, Curr. Biol. 10 (2000) R53-R55.

[19] N. Shibagaki, A.R. Grossman, The role of the STAS domain in the function and biogenesis of a sulfate transporter as probed by random mutagenesis, J. Biol. Chem. 281 (2006) 22964-22973.

[20] N. Rager, C.B. Mamoun, N.S. Carter, D.E. Goldberg, B. Ullman, Localization of the Plasmodium falciparum PfNT1 nucleoside transporter to the parasite plasma membrane, J. Biol. Chem. 276 (2001) 41095-41099. 